\title{
REVISIÓN DE LA ESTIMULACIÓN DEL PENSAMIENTO LÓGICO MEDIANTE EL PROGRAMA OBM EN EL RENDIMIENTO ESCOLAR DE LAS MATEMÁTICAS
}

\section{Dr. Luis Alberto Vicuña Peri}

La presente investigación tiene como propósito, verificar si el programa de estimulación para las operaciones lógicas básicas para el aprendizaje de la matemática aún tiene vigencia, puesto que la investigación original se realizo en 1983 con estudiantes del primer año de secundaria, de coeficiente intelectual normal, sin indicadores de lesión orgánica cerebral. En el presente, a partir que continúan las altas tasas de alumnos desaprobados en la asignatura de matemática, hemos creído pertinente efectuar una revisión del programa arriba mencionado pero esta vez ampliando la cobertura muestral, estudiantes del primero al quinto de secundaria, sin mayor control de variables que ser estudiante matriculado y en condiciones de regularidad. Esta modificación se hace con la intención de verificar si el manual funciona en las condiciones de aula donde los participantes varían en sus grados de homogeneidad. Para lo cual se les aplicó el test de operaciones clásicas para el aprendizaje de la matemática, que arroja cinco resultados: inclusión, clasificación, seriación, invariación e interpretación numérica además cuenta con un puntaje total, luego al grupo experimental se les aplicó sistemáticamente la estimulación y al termino se comprobó: primero que efectivamente las operaciones lógicas estimuladas se incrementan en comparación con el grupo control, y segundo, el grupo experimental que aumento su perfomance en las operaciones lógicas básicas incrementan significativamente el rendimiento escolar en la matemática.

PALABRAS CLAVE: Seriación, inclusión, clasificación, invariación, matemática

The present investigation has like intention, to verify if the program of stimulation for the basic operations logics for the learning of the mathematical one still has use, since the original investigation I am made in 1983 with students of the first year of secondary, of normal intellectual coefficient, without indicators of cerebral organic injury, the present to start off that continuous the high rates of students disapproved in the subjet of mathematical, we have believed pertinent, to carry out a revision of the program arrives mentioned but this time extending the cover sample, students of the first a fifth of secondary, without greater control of variables than to be registered student and in conditions of regularity, this modification it does with the intention to verify it the manual works in the conditions of classroom where the participants vary in their degrees of homogeneity. For which the test of basic operations for the learning of the mathematical one was applied to them, which it throws five results: inclusion, classification, serialization, invariacion and numerical interpretation in addition count on a record, soon to the experimental group the stimulation was applied to them systematically and to 1 finish was verified: first which indeed the operations stimulated logics are increased in comparison with the group control, and second, the experimental group that increase his perfomance in the basic operations logics significantly increases the scholastic yield in the mathematical one.

KEY WORDS: Serialization, inclusion, classification, invariacion, mathematical.

Colaborador: José Ysaías Ríos Díaz Miembro': Héctor Manuel Hernández Valz 


\section{INTRODUCCIÓN}

En el estudio realizado en 1983, Vicuña L. (2001) titulado «Repertorios disposicionales para el aprendizaje de las matemáticas en estudiantes del primer año de secundaria» se demostró, contrastando los rendimientos promedios de dos grupos de estudiantes diferenciados en cuanto a su performance en la asignatura de matemáticas pero igualados en CI, edad y sin indicadores de lesión orgánica cerebral, que diferían significativamente en las operaciones lógicas de: inclusión, clasificación, seriación e invariación, siendo los estudiantes de alto rendimiento en el curso de matemáticas lo que lograban mayores promedios; llegando a la conclusión que las operaciones lógicas mencionadas, actúan como repertorios básicos, sobre los cuales y en función a cómo se encuentren organizados en el sujeto ocurrirán o no nuevas asimilaciones que devengan en su ampliación cognitiva. Sobre esta conclusión, se consideró de suma necesidad, encontrar un medio práctico, sencillo y de alta eficiencia para estimular aquellas operaciones lógicas. Entre opciones como la estimulación directa individual, la estimulación grupal de tipo clase expositiva y diálogo de confrontación y en tercer lugar la estimulación mediante un texto programado nos decidimos por esta última opción ya que se aproxima al modelo: experimentador (E), sujeto (decodificador), R, experimentador (Ei) refuerzo, además presenta la ventaja que el ensayo puede repetirse tantas veces como sea necesario, sin el riesgo de introducir nuevos conceptos que pueden modificar al objetivo, así mismo se controlaron los posibles cambios de ánimo del experimentador que pudieron hacer variar las conductas de aproximación; pero tiene una desventaja, si el sujeto va más allá de lo que se ha planeado el texto puede resultar Iimitativo; esta desventaja la consideramos como posibilidad aún cuando en ninguno de los sujetos experimentales se haya presentado. Por otro lado, el texto puede ser utilizado por el mismo sujeto sin necesidad de un experimentador o instructor así mismo puede ofrecerse como lectura en las clases TUTORIALES o de APOYO COMPLEMENTARIO en los centros educativos, bondad que no podrá obtenerse con las otras opciones.

La presente investigación pretende aliviar la carencia de instrumentos válidos para la estimulación del pensamiento lógico, no sólo porque tenga una repercusión directa sobre el aprendizaje racional de las matemáticas sino también porque estimula al usuario: percibir y tratar la información en perspectiva, esto es de reconstruir mentalmente un hecho o fenómeno, decodificarlo desde el punto de vista del otro sin perder el suyo propio.

Al parecer, los diferentes medios que la era contemporánea ofrece al joven, tiende a mecanizarlo: las calculadoras de bolsillo le permiten alta eficiencia de cálculo; pero no el razonamiento lógico subyacente; los juegos y aparatos electrónicos computarizados se ofrecen a manera de un reto a las habilidades del joven; pero aquí también el sujeto desarrollará básicamente destreza en coordinación visomotriz. Inclusive se puede aprender el lenguaje de un computador y hacer programas simples siguiendo las pautas del modelo en una aparente lógica individual, cuando en sí está circunscrito dentro de los parámetros de la lógica del lenguaje que se utilice. Lo que perseguimos es que el hombre utilice estos medios para simplificar horas de trabajo, entendiéndolas como medios a su servicio y no como el solucionador de sus problemas, es decir, que el hombre vuelva los ojos hacia sí mismo y asuma que es él quien debe resolver los problemas y para ello se requiere que el individuo logre coronar su intelecto llegando a la lógica formal.

\section{PROBLEMA.}

Habiéndose demostrado en la investigación «Repertorios Disposicionales para el aprendizaje de las matemáticas en una muestra de estudiantes del Primero de secundaria», 
Vicuña, L (2001) que las operaciones lógicas de inclusión, seriación, clasificación e invariación influyen sobre el aprendizaje de las matemáticas; con estos datos creímos necesario averiguar qué pasa cuando se estimulan estas operaciones, Vicuña L. (1989. 1990) para tal fin en la mencionada investigación, trabajamos con una muestra de estudiantes varones del Primero de secundaria de CI promedio, cuyas edades cronológicas estaban comprendidas entre los 12.4 a 12.6 meses y dividido en un grupo control y otro experimental y formulamos las siguientes interrogantes:

¿Es suficiente la maduración cronológica, el avance escolar y el entorno medio ambiental o por el contrario es necesario estimular diferencialmente las operaciones lógicas para que los individuos lleguen a la reversibilidad proposicional del pensamiento lógico?

Al estimular las operaciones lógicas de: inclusión, seriación, clasificación e invariación ¿se afecta positivamente el rendimiento escolar de las matemáticas?

\section{HIPÓTESIS}

Se sustenta:

1. La maduración cronológica, el entorno medio-escolar-ambiental contribuyen en el desarrollo de las operaciones lógicas formando grupos heterogéneos, en tanto que, la estimulación diferencial logra grupos homogéneos asegurando que los sujetos lleguen a la lógica formal.

2. Al incrementarse el pensamiento lógico, los sujetos están en condiciones óptimas para tratar la informática conceptual de manera reversible 10 que debe facilitar la asimilación de las matemáticas y por lo tanto el incremento en el rendimiento en esta área del conocimiento.

El presente estudio tiene como propósito revisar los resultados que comprobaron las hipótesis mencionadas en 1989, pero esta vez no hemos controlado la inteligencia buscando que la composición de los participantes sea lo más aleatoria posible, con esto nuestra intención consiste en verificar si la estimulación es posible en condiciones académicas comunes, además con el mismo fin hemos ampliado el espectro muestral hasta el quinto año de secundaria, esperando además, que el programa de estimulación consolide las operaciones lógicas mencionada. Como se puede ver en el presente estudio volvemos a trabajar con los mismos problemas e hipótesis con la diferencia que no se controla inteligencia, y ampliamos el espectro muestral hasta el quinto grado de secundaria.

\section{Enfoque teórico}

Las múltiples interacciones del sujeto, desde su nacimiento, incrementan el proceso psicológico, que al parecer se inicia desde la etapa fetal, en un continuo cuyos límites resultan imposible establecer; ante esta realidad el trabajo científico de los psicólogos ha puesto especial énfasis en cómo se originan y desarrollan el pensamiento y lenguaje, la personalidad, la sensación, la percepción, el ajuste, la motivación, el aprendizaje y la manera como los factores sociales, madurativos, fisiológicos y patológicos interactúan afectándolas en alguna dirección.

En la presente investigación, nos interesa básicamente el pensamiento, no tanto para estudiar su formación y desarrollo como sí su permeabilidad para ser optimizado cuando se le estimula sistemáticamente; para lo cual trabajamos con los aportes del maestro J. Piaget, (1967) quien considera que la estructura del psiquismo es la función cognitiva, la que es dinamizada por la afectividad y ambas se expresan a través de la función de la representación. Donde el conocimiento está constituido por el pensamiento y la organización del la realidad; esto es el progresivo reemplazo de las acciones concretas por signos y 
símbolos que teniendo como base esquemas sensomotores se van distanciado hasta lograr conceptualizaciones hipotéticas proposicionales a un nivel estrictamente verbal.

Donde el pensamiento permite al individuo el tratamiento de la información: primero de manera mágica, intuitiva y transductiva, luego en forma simbólica e irreversible, posteriormente en forma reversible pero aún operatoria que es el paso hacia reversibilidad formal. Isomórficamente se construye la concepción del sí mismo y del universo respecto a las leyes que gobierna a la materia, tiempo y velocidad y sistemas numéricos. En esta continuidad evolutiva pondremos especial atención en el pensamiento lógico operativo; ya que es nuestra intención estimular las operaciones reversibles de manera tal que los sujetos lleguen a la lógica formal, asumimos, que las diferentes etapas en el desarrollo del pensamiento lógico y la organización de la realidad, no es únicamente función de la maduración biológica, sino además de la peculiaridad ambiental del sujeto que le ofrezca riqueza vivencial o por lo contrario niveles diferentes de carencia estimulativa; esto es que no basta tener 15 años o más para desenvolverse con propiedad lógica formal si es que el sujeto no ha: recibido un entrenamiento pertinente.

Es muy frecuente escuchar comentarios tales como «no razonan, no piensan, son mecanizados» y por lo general por parte de los profesores preferentemente de la asignatura de matemáticas y lógica tal vez reclaman un imposible si debiendo entrenar en razonamiento se limitan a la reproducción de paquetes cognitivos que deben ser resueltos según procedimientos ciegamente aplicados.

El escolar promedio se inicia hacia los seis años en la básica regular con objetivos muy concretos, deben aprender a leer y a escribir y algo de aritmética, básicamente a: contar y lograr correspondencias unívocas. Hacia los siete años, se sigue poniendo especial énfasis en la lecto-escritura y se incrementa la enseñanza de la aritmética en suma y resta de dos dígitos, hacia los ocho años y más, la enseñanza de las matemáticas imparte teorías de conjuntos y otras operaciones aritméticas con números naturales, enteros, fraccionarios y decimales. Al concluir este nivel hacia los doce años promedio, ingresa al nivel secundario, se inicia la enseñanza de las matemáticas con teoría de los conjuntos y luego ingresan al sistema numérico trabajando con números naturales y enteros en diversas funciones aritméticas y algebraicas; como se podrá apreciar en la enseñanza de teoría de conjuntos tanto en el nivel primario y retomado en la secundaria es muy adecuado; sin embargo hay un problema y que radica en que estos contenidos no cumplen sus objetivos básicos, esto es que el sujeto esté en capacidad para aplicar los principios en forma inductiva y deductiva; sino más bien se enfoca la atención a si el estudiante realiza o no una intersección o si es capaz de diagramar datos, donde es común que la tendencia del estudiante es fijar mnemónica mente los procedimientos y repetirlos mecánicamente; ¿por qué esta tendencia de los jóvenes estudiantes? Principalmente porque los contenidos se transmiten dando por establecido que ya cuentan con las condiciones de pensamiento básicas para asimilar y acomodar la nueva información, descuidando lo elemental, al no establecer en qué nivel de pensamiento lógico están y cuál es su performance en las operaciones de clasificación, inclusión, seriación e invariación. Este descuido lleva al estudiante a no decodificar la informática transmitida por el profesor y como se debe aprobar académicamente el curso, lo razonable y económico es utilizar la memoria fijadora y repetitiva. De esta manera, el pronóstico hacia una lógica formal estará condicionada a si ocurre o no el entrenamiento adecuado como también las diferencias individuales que de alguna manera logran decodificar la informática transmitida por sus profesores.

Hemos señalado que las condiciones básicas para un aprendizaje efectivo de las asignaturas que demandan del pensamiento lógico deberán estimular las operaciones que volvemos a mencionar: inclusión, seriación, clasificación e invariación que según lo hallado por J. Piaget, y confirmado en nuestro medio, Vicuña L (1989) durante la lógica 
operacional, estas operaciones están presentes y tienen características de reversibilidad, esto es, que los datos no sean interpretados en un solo sentido sino en su posición o parte relativa y que una operación pida ser anulada sin que sus unidades nocionales dejen de serlo; sin embargo estas operaciones sólo son posibles si los sujetos tienen la oportunidad de valerse de medios concretos en el aquí y ahora de los fenómenos físicos, esta capacidad de trascender hacia la lógica formal donde el pensamiento sea hipotético deductivo, pudiendo determinar lo real dentro del marco de lo posible, proposicional; donde ya no se manipulen datos directos de la realidad, sino afirmaciones que contienen esos datos y analítico combinatorio por el cual el sujeto incluye todas las combinaciones de las variables agotando el inventario posible.

En 1983, en una investigación para el Instituto de Investigaciones Psicológicas del Departamento de Psicología de la UNMSM titulada «Repertorios disposicionales para el Aprendizaje de las Matemáticas», Vicuña L. (2001) encontró que los estudiantes del $1^{0} \mathrm{de}$ secundaria, cuya edad promedio era de doce años, el promedio y sin indicadores de lesión orgánica cerebral, ubicados en el tramo final de la lógica operatoria e hipotética transición hacia la lógica formal, presentaban serios errores de pensamiento que los llevaba al fracaso en la resolución de problemas que exigen de inclusión, seriación, clasificación e invariación. Las respuestas erradas dejaban entrever irreversibilidad del pensamiento, tratando la información, no en un sistema integrado sino en subsistemas y que el fracaso era más notorio en aquellos estudiantes que al mismo tiempo presentaban bajo rendimiento en el curso de matemáticas. Llegando a la conclusión que estas operaciones lógicas, actúan como repertorios básicos para el óptimo aprendizaje de la matemática.

De lo hallado, surge la hipótesis que no basta el avance cronológico, la maduración pone al sujeto en estado potencial, la estimulación pertinente es necesaria tanto para la reversibilidad operatoria y más aún para la formal y que, si bien es cierto que los diferentes medios de comunicación social proporcionan en alguna medida pautas estimulativas, también es cierto que generan hábitos de pensamiento, basados en falacias, en generalizaciones de estímulo y de respuesta, restando al individuo la capacidad de análisis para pensar en perspectiva y que en la escolaridad, se traduce en mecanización de los aprendizajes, en la transferencia por similitud de estímulos antes que en la transferencia de principios que devienen en el fracaso en Ia matemática en altos porcentajes; hecho que se agrava cuando olvidamos que en el proceso madurativo, recién entre los 12 a los 15 años, los seres humanos hemos madurado neurofisiolgicamente al punto de pensar reversiblemente, el extremo inferior del rango cronológico señalado es la excepción, el promedio está alrededor de los 15 años; cuando el adolescente está por concluir la secundaria, aproximadamente está cursando el cuarto grado, de modo que solo le queda el quinto grado de secundaria para efectuar todo los ajustes reversibles a los conocimientos adquiridos durante toda su escolaridad, de forma mecánica repetitiva y no reflexiva, de modo que el joven concluirá la secundaria con un volumen de conocimientos no asimilados reflexivamente; por tanto se observa que los jóvenes completan su proceso madurativo matriculándose a las academias preuniversitarias para empezar a comprender lo que antes se les enseño pero que por las razones expuestas no tuvieron acceso. Esta vez sí tiene la potencialidad pero a su vez las academias han desarrollado técnica de marketing, ofertando el desarrollo de la comprensión verbal y el razonamiento numérico, convirtiendo a esta estrategia cognitiva en un conjunto de técnicas para solucionar problemas a partir de modelos tipo y no a partir del descubrimiento de principios transferibles proactiva y retroactivamente, de esta forma se va generando la idea que el éxito del individuo queda supeditado al éxito en la matemática, cayendo en lo absurdo cuando lo que debemos buscar es que el estudiante llegue al uso del pensamiento lógico formal como un medio para la autorrealización y con ello hacia hombres solucionadores activos de los problemas 
nacionales; y concurrentemente devendrá en el adecuado manejo de la matemática ya que este tipo de pensamiento es una condición básica para el éxito razonado de ésta asignatura.

Teniendo en cuenta la alta tasa de estudiantes que desaprueban podemos inferir que la probabilidad proporcional de sujetos que no llegan a la optimización de la lógica formal también será alta y con ello el panorama del hombre solucionador de problemas se torna sombrío. De lo descrito, resulta conveniente y necesario, desarrollar programas que contribuyan a que el pensamiento lógico formal sea una realidad pata el mayor número de personas.

El presente estudio, pretende contribuir reduciendo esta necesidad para lo cual revisamos la validez del programa, elaborado y experimentando con una muestra de estudiantes de doce años de edad, que cursaban el primero de secundaria, igualados en inteligencia replicando el estudio pero esta vez con muestras transversales aleatoriamente elegidos con el propósito de conducirlos hacia la asimilación de información, partiendo del aquí y ahora de los fenómenos concretos, realizando manipulaciones de datos, primero al mismo nivel, para luego sistemáticamente vayan ingresando al trabajo hipotético, proposicional y combinatorio en lo que respecta a las operaciones: de clasificación, inclusión, seriación e invariación, que asumimos son susceptibles de ser estimulados y por ende incrementarse significativamente a partir de los doce años asegurando el ingreso hacia la lógica formal.

\section{METODOLOGÍA}

El presente estudio cuasi experimental de análisis de momentos con grupo de control, se desarrolló con una muestra transversal constituida por cinco grupos a uno por cada grado escalar del nivel secundario, la muestra se subdividió al azar en dos subgrupos, uno de ellos sometidos a estimulación, el otro dejado a su desenvolvimiento natural.

Para la estimulación, se elaboró un manual programado dividido en cuatro capítulos: Clasificación, Seriación, Inclusión e Invariación. Cada capítulo está graduado para 45 minutos de lectura que se presentaron en dos sesiones distintas dentro de la misma semana, al concluir cada capítulo se utilizaron dos sesiones de 45 minutos cada una, para evaluar, mediante el diálogo el grado de asimilación del material y si se transfería a nuevas situaciones. La estimulación fue de la siguiente manera, primero los participantes en cada sesión leen el manual, una vez terminado se discute y se ponen ejemplos basado en el tema, el tiempo de lectura es de 40 minutos y la discusión dinámica es de 15 minutos. Este esquema es constante para cada una de las cuatro operaciones que se estimulan: clasificación, inclusión, seriación e invariación. Concluido las cuatro sesiones se descansa por un periodo de diez días al termino de los cuales se repite el mismo procedimiento.

La elección de la muestra fue bietápica, en primer lugar se procedió a buscar un centro educativo que además de mostrar interés, diera las facilidades para efectuar las evaluaciones pre y post estimulación, y sobre todo el ambiente apropiado para la estimulación o fase experimental, de esta manera se seleccionó un Centro educativo de varones del nivel secundario y de gestión estatal de la Ciudad de Lima, durante el periodo lectivo del año 2002, la segunda etapa consintió en elegir del total de estudiantes que cursaban la secundaria del primero al quinto a 50 alumnos de cada grado donde la mitad sería grupo control y la otra mitad experimental, el tamaño de muestra se decidió teniendo en cuenta, el número de personas con las que se puede trabajar en grupo sin perder el control debido al anonimato, que ocurre cuando los grupos de trabajo son más de 25 , quedando una muestra de 250 estudiantes, la elección de cada cincuenta por grado y de cada 25 por subgrupo se realizo con el método aleatorio simple con remplazamiento.

A la muestra elegida se le aplicó el Test de Operaciones Básicas Para el Aprendizaje de la Matemática, Vicuña Luis, (2001) en el momento inicial. El mencionado test evalúa cinco operaciones: seriación, inclusión, interpretación numérica, clasificación e invariación, y un 
puntaje total: la confiabilidad para los subtests con el método de las mitades van desde un mínimo de 0.81 hasta 0.94 y para el test total 0.86 , por el método de la consistencia interna los coeficientes van desde 0.86 hasta 0.90 y para el test total 0.90 . La validez es de constructo y predictiva en la primera se confirma la presencia de cinco factores y por la segunda arroja un coeficiente de 0.60 con el rendimiento escolar de la matemática. En cuanto a los itemes estos fueron rigurosamente seleccionados en base al índice de dificultad comprendidos entre 0.40 hasta 0.60 .

Para el rendimiento escolar de la matemática se utilizo los calificativos de los exámenes escritos correspondientes al segundo y tercer bimestre.

El procesamiento de datos fue mediante el análisis de estadísticos descriptivos e inferenciales, para precisar los atributos cuantitativos de las variables estudiadas y el riesgo de error probable de las conclusiones, posibilitando el análisis cualitativo en la demostración de las hipótesis.

\section{ANÁLISIS DE RESULTADOS}

Empezaremos describiendo a la muestra dividida en grupo control y experimental en el momento previo a la estimulación, hemos encontrado que no difieren en ninguna de las operaciones lógicas, sin embargo es conveniente señalar que los participantes que pertenecen al primer y segundo grado son muy similares en las puntuaciones obtenidas, y que resulta diferente al tercer grado y éstos configuran un grupo diferente a los jóvenes que cursan el cuarto y quinto grado de secundaria, las edades correspondientes van el primer bloque tienen 12 a 13 años seis meses. El segundo bloque corresponde 14 años hasta 14 años seis meses y el tercer bloque esta dado por jóvenes cuya edad promedio es de 15 años 8 meses. Como se puede ver en la tabla que a continuación se reproduce, el pensamiento lógico operatorio pasa por tres estadios antes de llegar a la lógica formal: el primer estadio corresponde al primero y segundo grados, el segundo estadio al tercer grado y el tercero al cuarto y quinto grados de secundaria. Esto por las diferencias observadas; para efectos de nuestro estudio vemos que los participantes como grupo control y grupo experimental, proceden de una misma población, conclusión a la que se llega al no encontrar diferencias entre ambos grupos dentro de cada grado escolar. 
Tabla Nro. 01. De comparaciones de medias aritméticas de las operaciones básicas para el aprendizaje de la matemática en función al grupo control y experimental para los cinco grados de educación secundaria, en el momento inicial.

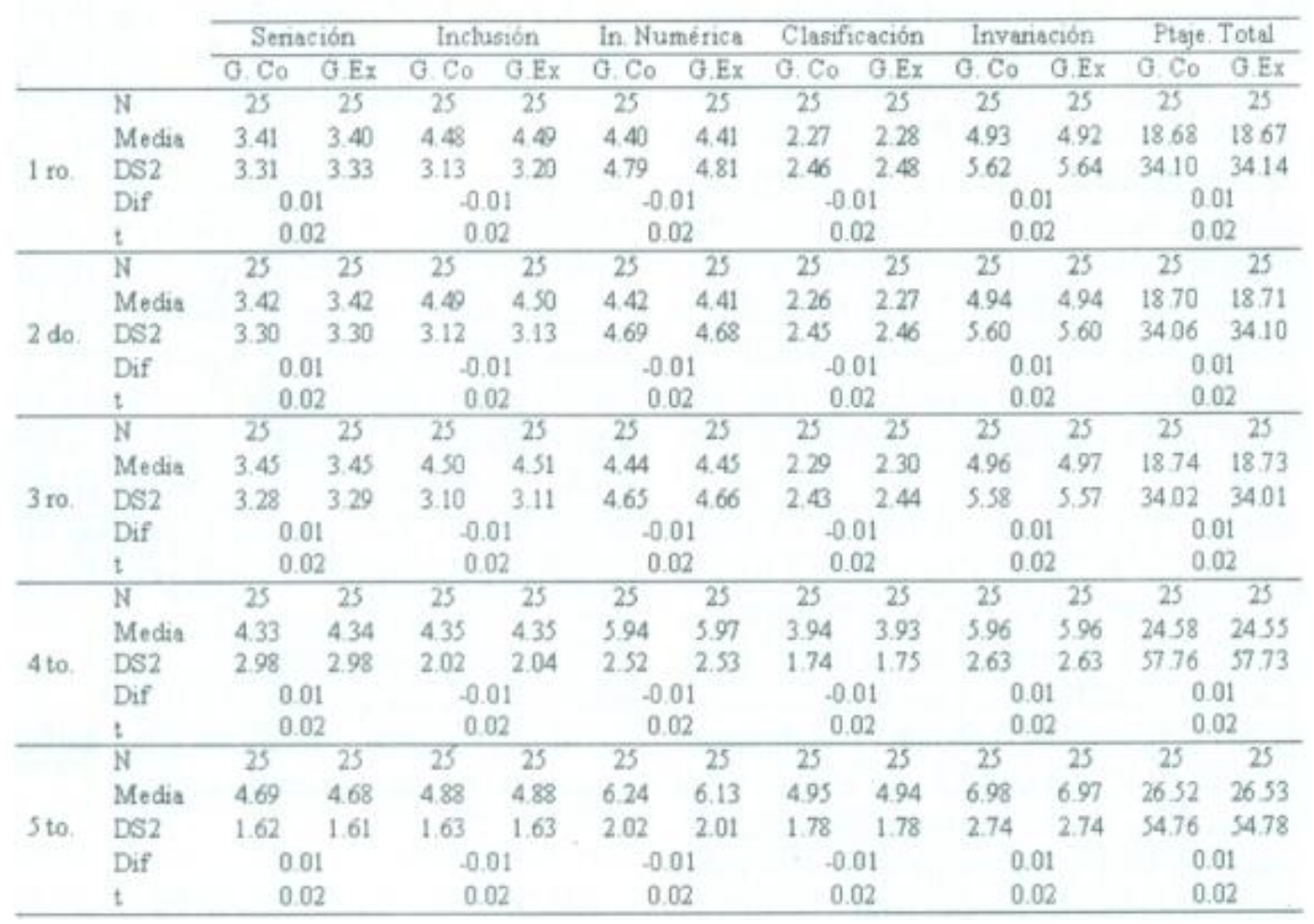

Para analizar el papel que cumple la estimulación de las operaciones lógicas, el grupo experimental fue estimulado durante 2 meses en las operaciones lógicas de clasificación, inclusión seriación e invariación; no se estimuló interpretación numérica porque de alguna manera es estimulada en el curso de matemáticas para toda la población escolar. Por su parte, el grupo control, con características similares al grupo experimental fue observado en su desenvolvimiento natural. Nueve meses después del examen de entrada se retestó a ambos grupos y los resultados se contrastaron entre el momento 1 con el momento 2 ; intragrupalmente, y el mismo análisis extragrupalmente. Al contrastar los resultados extragrupalmente, se espera encontrar diferencias significativas entre los sujetos estimulados y los de control; para afirmar que la estimulación ha cumplido su propósito, incrementando las operaciones lógicas en los sujetos experimentales respecto a los sujetos del grupo control. En las tablas 2 y 3 que a continuación se reproduce contrastamos los resultados del retest tanto en el grupo control como en el experimental, encontrando que los participantes del programa estimulativo, incrementan significativamente su performance respecto al grupo control en todos los grados escolares. También se puede ver que las condiciones de estimulación medio ambientales, producen incrementos espurios o irrelevantes, dejando entrever que el papel estimulativo, de la comunidad y el colegio no es suficiente siendo necesaria la aplicación de programas que estimulen diferencialmente para asegurar el tránsito hacia la lógica formal. 


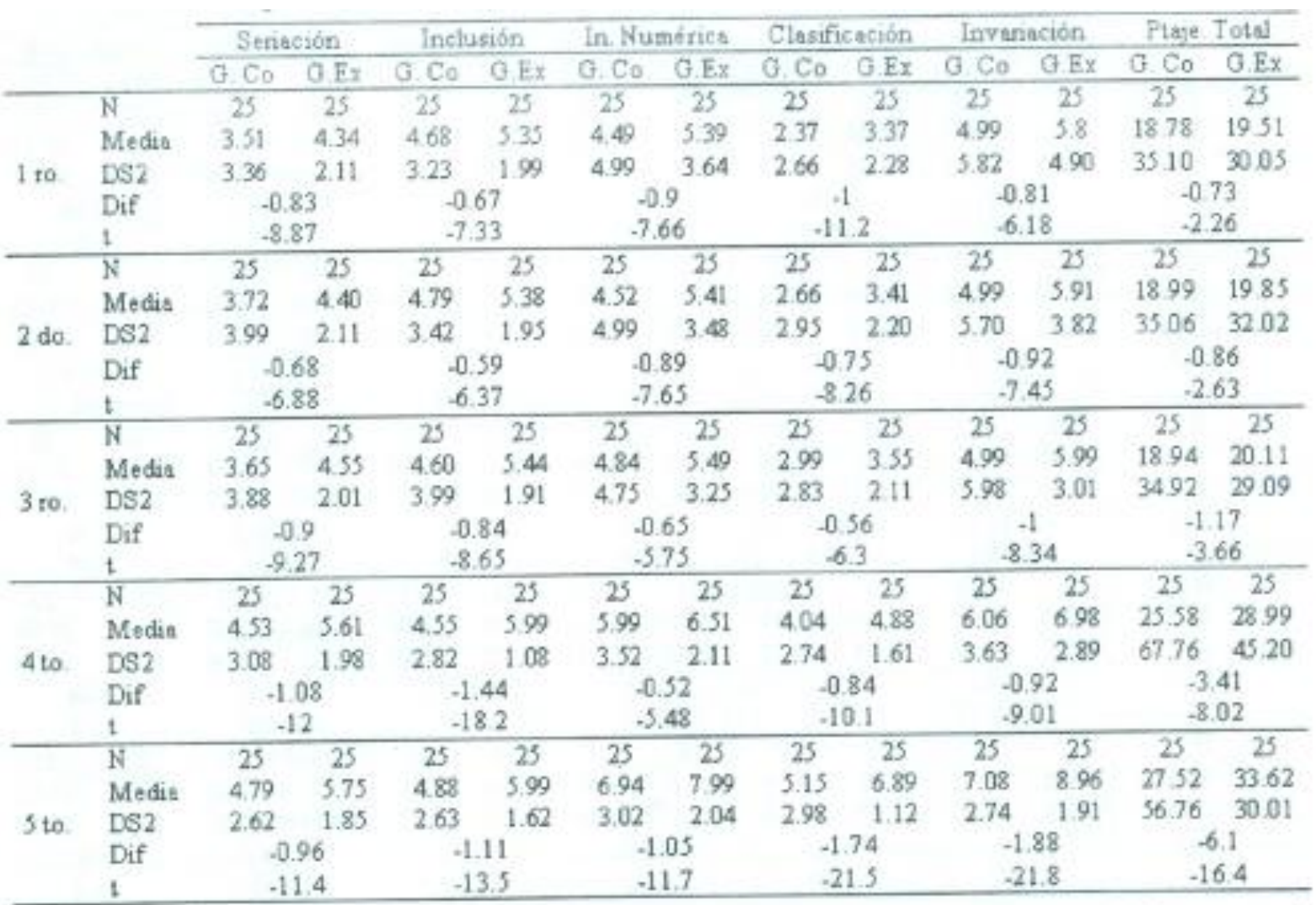

Tabla Nro 03. De comparaciones de medias aritméticas de las operaciones básicas para el aprendizaje de la matemática correspondiente al grupo control en los momentos antes y después, en los cinco grados de educación secundaria.

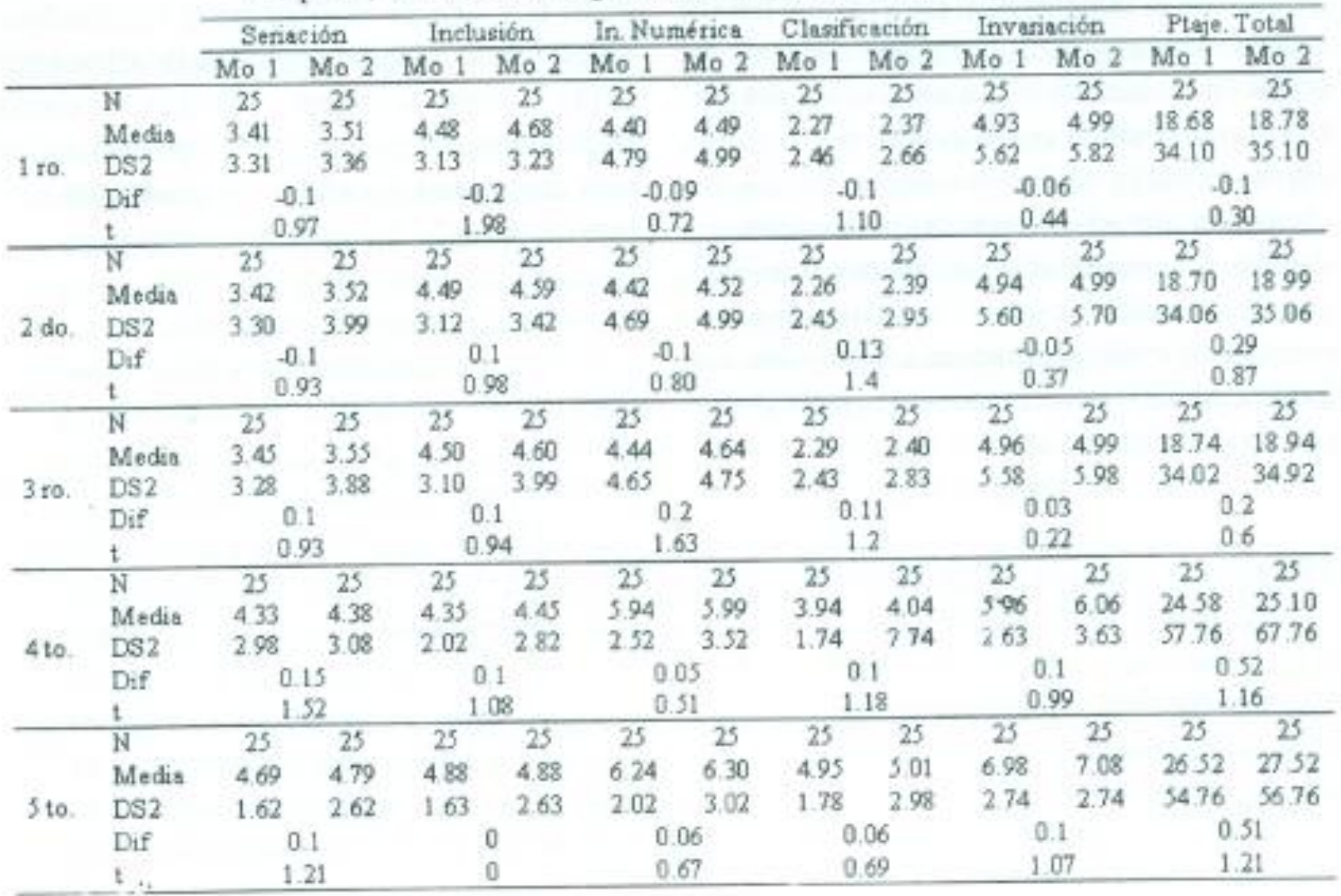


Tabla Nro 4. De comparaciones de medias aritméticas de las operaciones básicas para el aprendizaje de la matemática correspondiente al grupo experimental en los momentos antes y después de la estimulación, en los cinco grados de educación secundaria.

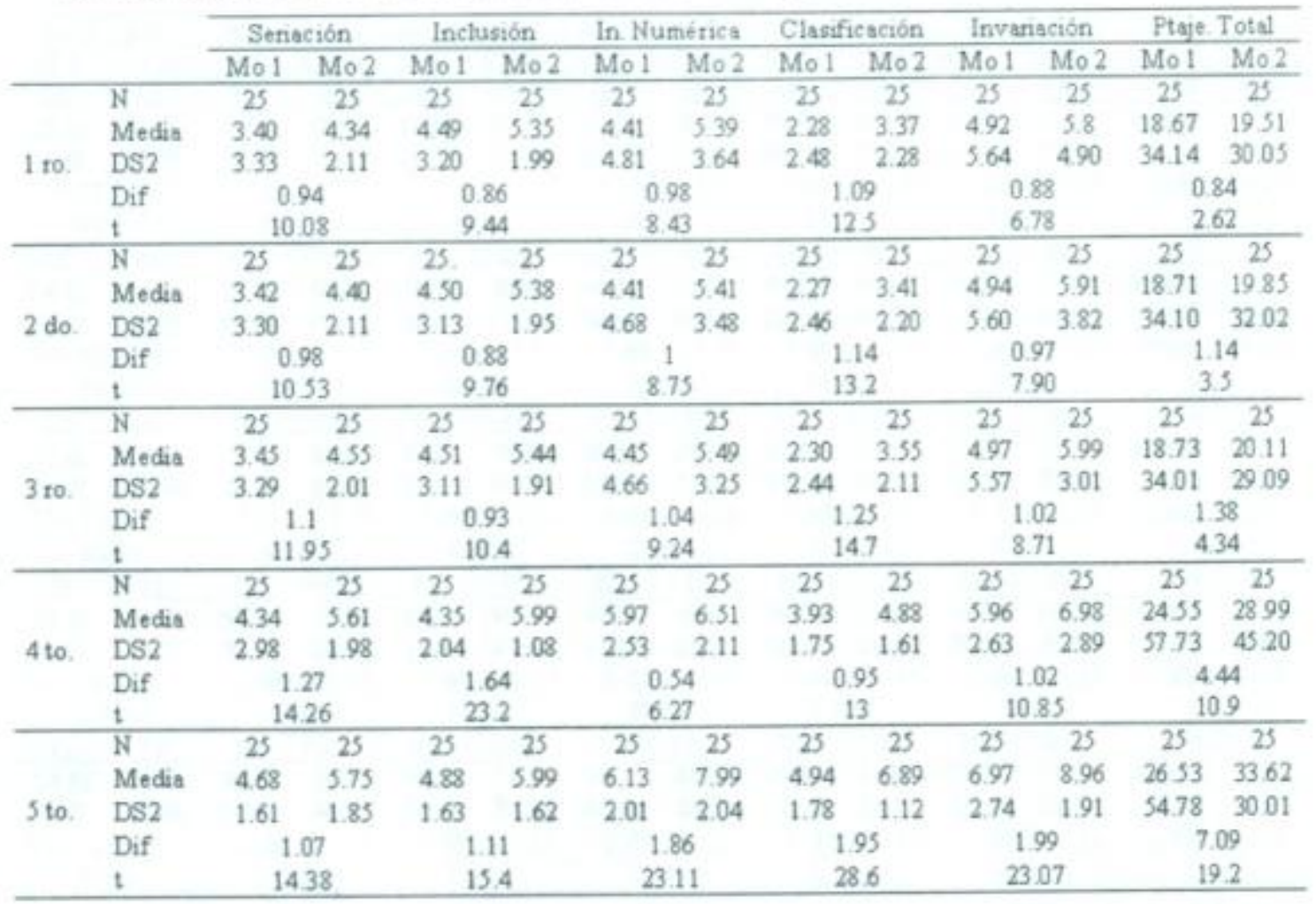

Cuando observamos el papel que cumplen estas operaciones sobre el rendimiento académico de la matemática, para lo cual hemos contrastado mediante la razón crítica de diferencias de medias las puntuaciones del curso de matemática correspondiente al grupo experimental y al grupo control, hemos encontrado que el primer grupo incrementa significativamente el rendimiento promedio, en comparación al grupo control, cuyos incrementos entre el momento uno y dos son irrelevantes.

De lo hallado se desprende que al incrementar la perfomance en las operaciones lógicas también se incrementa el rendimiento escolar de la matemática lo que confirma que estas operaciones actúan como disposicionales para el óptimo aprendizaje de esta asignatura, en todos los grados escolares. 
Tabla $\mathbf{N}^{\circ} 5$ de Medidas de tendencia central y de comparación de medias aritméticas del rendimiento escolar de la matemática en los momentos pre y post estimulación en el grupo control y experimental de los cinco grados de instrucción secundaria.

\begin{tabular}{|c|c|c|c|c|c|}
\hline & & \multicolumn{2}{|c|}{ Grupo Control } & \multicolumn{2}{|c|}{ Grupo Experimental } \\
\hline & & Momento 1 & Momento 2 & Momento I & Momento 2 \\
\hline \multirow{6}{*}{ ler. Grado } & $\mathrm{N}$ & 25 & 25 & 25 & 25 \\
\hline & Media & 12.35 & 1232 & 12.45 & 13.21 \\
\hline & Mediana & 12 & 12 & 12 & 13 \\
\hline & DS2 & 9.42 & 9.41 & 9.85 & 4.22 \\
\hline & Diferencia & \multirow{2}{*}{\multicolumn{2}{|c|}{$\begin{array}{l}0.03 \\
0.17 \\
\end{array}$}} & \multirow{2}{*}{\multicolumn{2}{|c|}{$\begin{array}{l}0.76 \\
5.07\end{array}$}} \\
\hline & $t$ & & & & \\
\hline \multirow{6}{*}{2 do grado } & $\mathrm{N}$ & 25 & 25 & 25 & 25 \\
\hline & Media & 12.28 & 12.30 & 12.31 & 13.08 \\
\hline & Mediana & 12 & 12 & 12 & 13 \\
\hline & DS2 & 9.23 & 9.22 & 9.65 & 4.08 \\
\hline & Diferencia & \multirow{2}{*}{\multicolumn{2}{|c|}{$\begin{array}{l}0.02 \\
0.12\end{array}$}} & \multirow{2}{*}{\multicolumn{2}{|c|}{$\begin{array}{c}0.77 \\
5.2 \\
\end{array}$}} \\
\hline & $t$ & & & & \\
\hline \multirow{6}{*}{3 ef grado } & N & 25 & 25 & 25 & 25 \\
\hline & Media & 11.89 & 1185 & 12.01 & 12.98 \\
\hline & Mediana & 12 & 12 & 12 & 13 \\
\hline & DS2 & 9.78 & 9.77 & 9.99 & 4.89 \\
\hline & Diferencia & \multirow{2}{*}{\multicolumn{2}{|c|}{$\begin{array}{l}0.04 \\
0.23\end{array}$}} & \multirow{2}{*}{\multicolumn{2}{|c|}{$\begin{array}{l}0.97 \\
6.29\end{array}$}} \\
\hline & $t$ & & & & \\
\hline \multirow{6}{*}{4 to. grado } & $\mathrm{N}$ & 25 & 25 & 25 & 25 \\
\hline & Media & 1196 & 1198 & 12.05 & 13.06 \\
\hline & Mediana & 12 & 12 & 12 & 13 \\
\hline & DS2 & 9.0 & 9.01 & 9.45 & 4.6 \\
\hline & Diferencia & \multirow{2}{*}{\multicolumn{2}{|c|}{$\begin{array}{l}0.02 \\
0.12\end{array}$}} & & \\
\hline & $t$ & & & \multicolumn{2}{|c|}{6.74} \\
\hline \multirow{6}{*}{5 to grado } & N & 25 & 25 & 25 & 25 \\
\hline & Media & 12.21 & 12.20 & 1235 & 13.28 \\
\hline & Mediena & 12 & 12 & 12 & 13 \\
\hline & DS2 & 8.45 & 8.39 & 9.05 & 4.8 \\
\hline & Diferencia & \multirow{2}{*}{\multicolumn{2}{|c|}{$\begin{array}{l}0.01 \\
0.06\end{array}$}} & \multirow{2}{*}{\multicolumn{2}{|c|}{$\begin{array}{l}0.93 \\
6.25\end{array}$}} \\
\hline & $t$ & & & & \\
\hline
\end{tabular}

\section{CONCLUSIONES}

1.- Si bien la evolución cronológica y las múltiples interacciones medioambientales actúan de alguna manera para que el individuo desarrolle estructuras lógicas formales, éstas no son suficientes requiriéndose de una estimulación adicional, orientada específicamente a la adquisición de nuevas asimilaciones que consoliden la ampliación de los esquemas básicos adquiridos, de no ser así, tendremos grupos humanos muy heterogéneos, unos llegarán a permanecer dentro de un sentido común, apoyado en la intuición, en la transferencia de generalizaciones y de transposiciones muy distantes de un pensamiento proposicional, hipotético y combinatorio, siendo sujetos que tienden a los dogmas, a la reversibilidad de los hechos y fenómenos dentro de los procesos que implican continuidad. Lo dicho se ratifica al encontrar que en la escolaridad no sólo las matemáticas arrojan un alto índice de dificultad, sino también las asignaturas de lógica, cuya dificultad no sólo alcanza a la secundaria sino que se extiende aún hasta loa ámbitos de la educación superior.

2.- La operaciones lógicas son susceptibles a ser estimuladas a partir de los 12 años de edad como se ha comprobado en la presente investigación. Su estimulación conduce a los participantes hacia la coronación del intelecto llegando a las estructuras lógica formal.

3.- El incremento de la performance en las operaciones lógicas de seriación, inclusión, invariación y clasificación, capacitación al estudiante para lograr mejores asimilaciones 
que de vendrán en acomodación cognoscitivas pertinentes a toda información que requiere de un análisis proposicional, hipotético y combinatorio como es la ciencia física matemática, las ciencias naturales y el pensamiento en perspectiva hacia las ciencias sociales.

\section{RECOMENDACIONES}

1.- Es conveniente que los profesores de matemáticas antes de poner énfasis en las enseñanzas de los conocimientos en torno a leyes, principios y aplicaciones que a menudo se tornan mecánicos; pongan especial atención en el momento de proposiciones, formulación de problemas, sustentación de hipótesis y métodos para su demostración, donde un mismo hecho o fenómeno se aplicado en su aco!1tecimiento relativo y contrastado en circunstancias diferentes. Que las leyes y principios no sean dogmas, sino más bien como recursos que sirven para explicar en función de los parámetros hallados y la susceptibilidad de cambio al manipularse los eventos.

2.- Construir y difundir mediante los diferentes medio de comunicación lógico con el propósito de asegurar que los individuos adquieran mejores repertorios, que constituyan en los esquemas básicos sobre los cuales puedan ocurrir mejores acomodaciones asegurándonos del desarrollo del intelecto hacia una optimización del potencial psíquico, que se traducirá en mayor producción y productividad al servicio de la nación.

\section{REFERENCIAS BIBLIOGRAFÍAS}

Piaget J. Szeminska. (1967): Génesis del número en el niño. Buenos Aires Guadalupe.

Deterline W. (1969): Introducción a la enseñanza programada. Buenos Aire - Troquel.

Vicuña L. (1989) Efectos de la

Estimulaciones de los Repertorios Disposicionales sobre el rendimiento escolar de las matemáticas en estudiantes del primero de secundaria del Colegio Aplicación San Marcos. CEDEIS. Año II W 5.

Vicuña L. (1990) Efectos de la Estimulaciones de los Repertorios Disposicionales sobre el rendimiento escolar de las matemáticas en estudiantes del primero de secundaria del Colegio Aplicación San Marcos. CEDEIS 1990. º 6.

Vicuña L. (2002) Test de Repertorios Básicos para el aprendizaje de la matemática. Año 1. Vól. Nro.1 y 2 PARADIGMAS. Colegio de Psicólogos del Perú. 